\title{
Giant Mesenteric Hemangioma without Small Intestinal Involvement Report of a Case and Review of Literature
}

\author{
Jui-Ten Wu, Chun-I Tsai, and Swei-Hsiung Tsung
}

\section{ABSTRACT}

Hemangioma involving the mesentery was very uncommon. Up to date, there were approximately twenty cases reported in the English literature, of which five cases were designated as giant hemangioma. We reported another giant mesenteric hemangioma, measuring $35 \times 20 \times 11 \mathrm{~cm}$. with a weight of 5,5 Kilograms. The etiology of mesenteric hemangioma was still debatable. In our case, the patient only experienced mild abdominal pain, without gastrointestinal hemorrhage. Therefore, we were in favor of mesenteric origin as opposed to gastrointestinal origin. The symptoms were non-specific, ranging from abdominal pain, nausea, vomiting, and gastrointestinal bleeding if Gastrointestinal tract was involved. Preoperative diagnosis was very challenging despite the modern images' technics have become available, such as computed tomography magnetic resonance and ultrasonography. The final diagnoses of mesenteric hemangioma have been exclusively established by histological examination after surgical removal of the tumor. Surgical resection with clear margin is the treatment of choice.

Keywords: Abdominal pain, Capillary, Cavernous, Gastrointestinal tract, Hemangioma, Mesentery, Surgical removal.
Submitted : July 07, 2021

Published : July 25, 2021

ISSN: 2593-8339

DOI: $10.24018 /$ ejmed.2021.3.4.968

Jui-Ten Wu

Director, Department of Surgery, St Mary's Hospital, Loudong, Yilan, Taiwan.

Chun-I Tsai

Director, Chun-I Tsai GI Clinic, Yilan, Taiwan.

Swei-Hsiung Tsung*

Director, Department of Pathology, St Mary's Hospital, Loudong, Yilan, Taiwan.

(e-mail: tsung.sweihsiung @ ${ }^{\circledR}$ gmail.com)

*Corresponding Author

\section{INTRODUCTION}

Hemangiomas are one of the most common soft tissue benign neoplasms. They can arise everywhere in almost any organs in the body. However, hemangioma involving the mesentery is very uncommon. Up to date, there were approximately twenty cases reported in the English literature [1], of which five cases were designated as giant hemangioma [1]-[5]. Among the five cases their measurement ranged from $14 \times 17 \times 7 \mathrm{~cm}$ to $36 \times 33 \times 8 \mathrm{~cm}$. Herein, we report additional giant mesenteric Hemangioma, measuring $35 \times 20 \times 11 \mathrm{~cm}$. with a weight of $5,5 \mathrm{~kg}$.

\section{CASE REPORT}

A 46-year man sought medical attention because of mild abdominal pain for two weeks. On physical examination, his abdomen was slightly distended. No tumor mass was palpable. Laboratory data were within normal limits except for slight anemia, with Hemoglobin value of $9.5 \mathrm{~g} / \mathrm{L}$. Gastroscopic examination was done, and mild chronic gastritis was found. Ultrasonography revealed a large cystic mass (Fig. 1 A). It was worthy mention that tumor markers including serum levels of CEA, CA19-9 and alphafetoprotein were all within normal limits. With the diagnosis in doubt, the patient was referred to St Mary's Hospital for further evaluation. Without delay, computed tomography (CT) with and without contrast media was performed. Both demonstrated a heterogeneous giant cystic mass (without enhancement) (Fig. 1 B). Because of the size of the tumor, an open surgery was planned. After the surgeon opened the abdomen, a large tumor originated from the mesentery was seen. This large mass was tightly adhered to a $30 \mathrm{~cm}$ segment of ileum which was resected with the tumor. After the resection was done, multiple adhesions were identified between the cyst wall and the organs such as transverse colon, liver, duodenum, and gallbladder. Adhesions were detached, and the mesenteric tumor was removed en bloc.

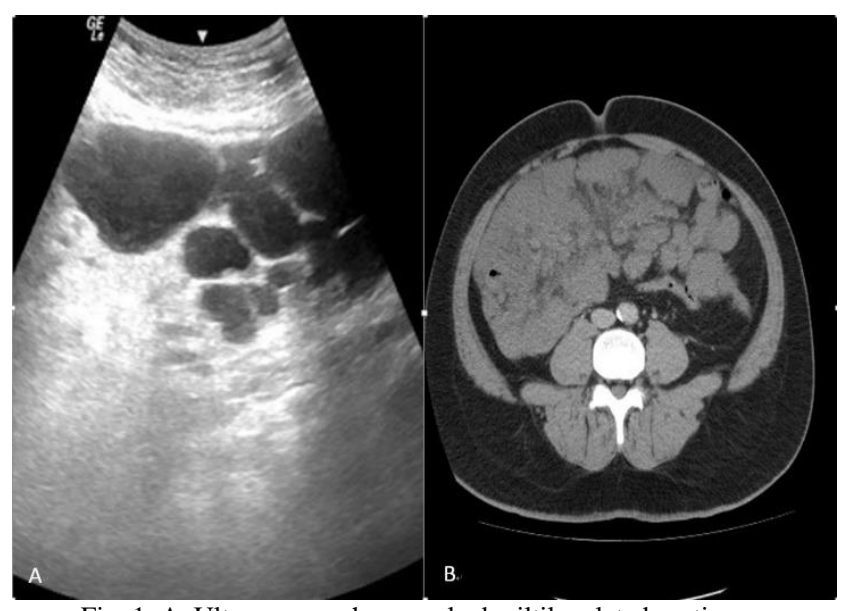

Fig. 1. A. Ultrasonography reveal ed miltiloculated cystic mass. B.Computed tomography showed a heterogeneous mass. 


\section{PATH Histology}

The external appearance of the tumor was reddish, convoluted and focally hemorrhagic, measuring $35 \times 20 \times 11 \mathrm{~cm}$, with a weight of $5.5 \mathrm{~kg}$ (Fig. $2 \mathrm{~A}$ ) The cut surface was multiloculated cystic, with honeycomb appearance (Fig. 2 C). A segment of ileum $30 \mathrm{~cm}$ in length, and $4 \mathrm{~cm}$ in circumference was firmly attached to the tumor which was resected. On opening, the lumen of the ileum was empty, and the mucosa showed normal folds without evidence of ulceration (Fig. 2 B). Histologically, the tumor was composed of proliferating capillaries (Fig. 3 A) admixed with cavernous veins (Fig. 3 B). No extension to the ileum was noticed. On Immunohistochemical stain. The tumor was positive for CD 31 (Fig. 3 C) and CD 34 (Fig. 3 $\mathrm{D})$, which are specific markers for hemangioma. Therefore, the diagnosis of giant cystic hemangioma in mesentery was rendered.
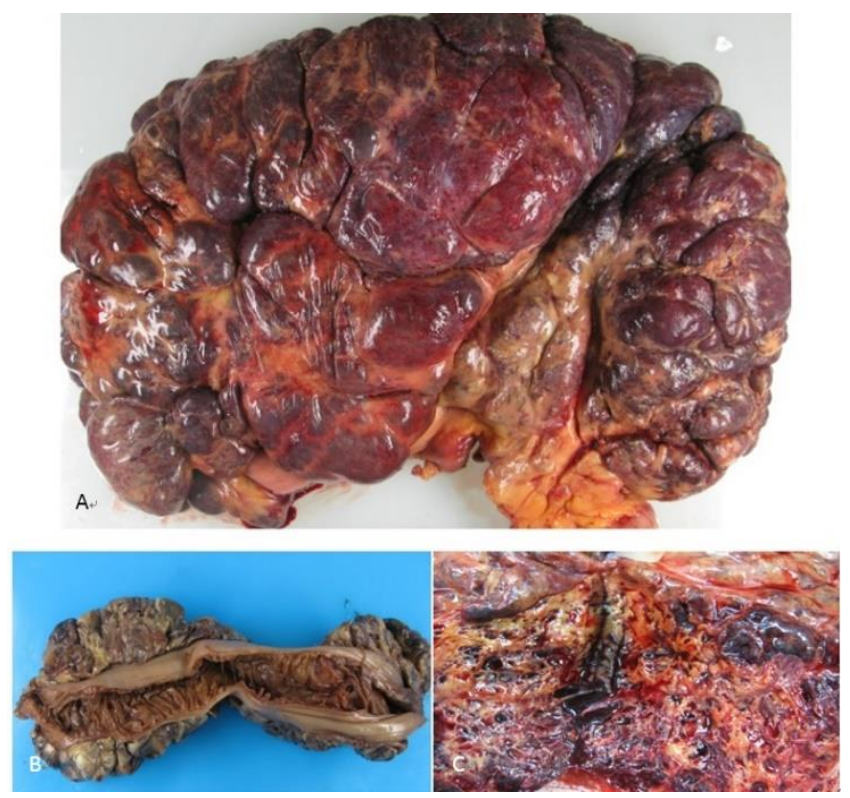

Fig. 2. A. The mesenteric hemangioma, with convoluted surface. B. Attached to the tumor was a segment of ileum with normal mucosa. C. The cut surface of the tumordiplayed a honeycomb appearance with foci of hemorrhage.

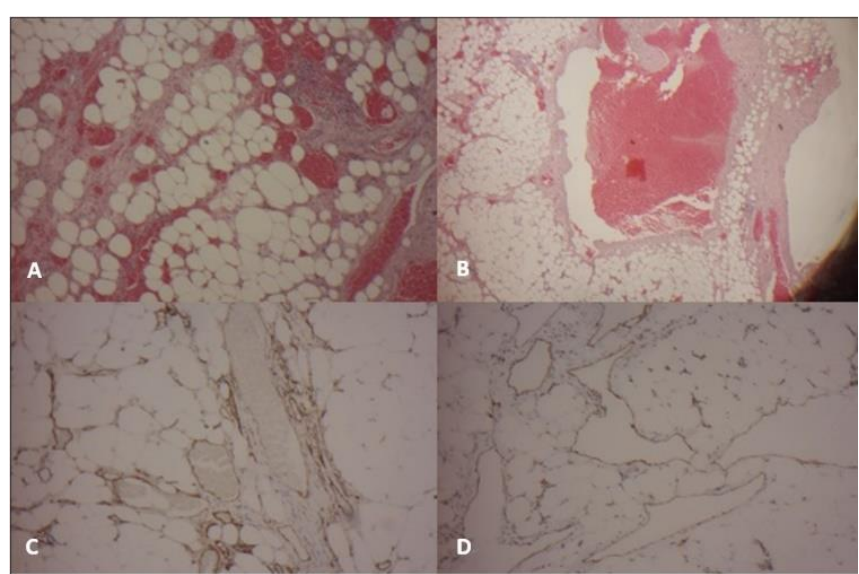

Fig. 3. Histopathology. A. Proliferation of capillaries engorged with erythrocytes in their lumina. $\mathrm{H}$ and $\mathrm{E}$ stain $\times 200$. B. Cavernous vein showing dilatation of its lumen and filled with RBC. H and E stain, $\times 200$.

\section{DISCUSSION}

In histopathology, there are three types of hemangioma [6] 1) capillary type; 2) cavernous type; 3) capillary and cavernous mixed type. Among which, the most common one was cavernous type, characterized by a dilated space filled with red blood cells and lined by a single layer of endothelial cells. Our case fit type three well.

The origin of mesenteric hemangioma is still debatable. In some reported cases and ours, the tumors were limited in the mesentery rather than the bowel wall that made us believe that the hemangioma was originated from the mesentery. The symptoms of mesenteric hemangioma were not specific ranging from mild to severe abdominal pain, nausea, and vomiting. If the hemangioma involved the intestine, and eroded the mucosa, the patients may present gastrointestinal bleeding. In our case, the bowel structure was reserved, so no bleeding was observed. However, insidious bleeding from the capillaries into the peritoneum resulted in mild anemia. Less commonly, if the mesenteric hemangioma is associated with intestinal polyps, intestinal intussusception may occur. Malignant transformation has been exceptional [7].

\section{CONCLUSION}

We reported an uncommon case of giant mesenteric hemangioma which could occur in young child or adult. Its symptoms were non-specific or variable. Even with modern advanced imaging techniques, the preoperative diagnosis is difficult, if not impossible. The treatment of choice is surgery. If the tumor is too large to be excised, low-dose radiation therapy, cryotherapy, brachytherapy, or arterial embolization has been used with limited success [8], [9].

\section{REFERENCES}

[1] Yang CZ, GZ, Li J, Jin H. Giant mesenteric hemangioma of cavernous and venous mixed type: a rare case report. BMC Surg, 2013:50-54.

[2] Conroy CF, Tokuso T. Giant cavernous hemangioma of the mesentery. Am J Surg, 1951:82(2):293-4.

[3] Rathnaraj S, Aggarwal S, Verghese M. Giant mesenteric hemangioma. Indian J of Gastroenterolgy, 1995:14(3):112.

[4] Ruiz AR, Ginsberg AL. Giant mesenteric Hemangioma with small intestinal involvement. Dig Dis Sci., 1999;44:(12):2545-2551.

[5] Baek SJ, Shin JW, Kim J. An extremely rare case of giant mesenteric hemangioma. ANZ J Surg, 2013;83(6):487-488.

[6] Abrahamson A, Shandling B: Intestinal hemangghioma in childhood and a syndrome for Diagnosis. A collective review. J Pediatr Surg, 8:487-495, 1973.

[7] Murray-Lyon IM, Doyle G. Porter N. Hemangiomatosisof the small and large bowel with histological malignant change. J Pathol, 105:295-297.

[8] Allred HW, Hemangioma of the colon, rectum, and anus. Mayo Clin Proc, 1974, 9:739.

[9] Lyon D, Mantia A: Large bowel hemangioma. Dis Colon Rectum., 1987. 27:404-14. 